\title{
Dose-Dependent Influence of Antithyroid Drugs on the Difference in Free Thyroxine Levels between Mothers with Graves' Hyperthyroidism and Their Neonates
}

\author{
Hiroyuki Iwaki $^{a}$ Kenji Ohbab Eisaku Okadac Takeshi Murakoshid \\ Yumiko Kashiwabara ${ }^{a}$ Chiga Hayashia Akio Matsushita ${ }^{\mathrm{e}}$ Shigekazu Sasaki ${ }^{\mathrm{a}}$ \\ Takafumi Suda ${ }^{\text {e }}$ Yutaka Oki ${ }^{\mathrm{f}}$ Rieko Gemma ${ }^{a}$ \\ a Division of Endocrinology, Department of Internal Medicine, Seirei Hamamatsu General Hospital, Hamamatsu, \\ Japan; bedical Education Center, Hamamatsu University School of Medicine, Hamamatsu, Japan; ' Department \\ of Community Health and Preventive Medicine, Hamamatsu University School of Medicine, Hamamatsu, Japan; \\ ${ }^{d}$ Obstetrics and Gynecology, Maternal and Perinatal Care Center, Seirei Hamamatsu General Hospital, Hamamatsu, \\ Japan; 'Second Division, Department of Internal Medicine, Hamamatsu University School of Medicine, Hamamatsu, \\ Japan; ${ }^{\mathrm{f}}$ Department of Metabolism and Endocrinology, Hamamatsu-Kita Hospital, Hamamatsu, Japan
}

\section{Keywords}

Antithyroid drugs · Graves' disease · Neonatal thyroid dysfunction · Pregnancy · Thyroxine

\begin{abstract}
Background: Several guidelines have recommended that the use of the lowest effective dose of antithyroid drugs (ATDs) that maintains maternal serum free thyroxine (FT4) levels at or moderately above the upper limit of the reference range is appropriate for fetal euthyroid status. However, little is known about whether ATD dosage affects the difference in serum FT4 levels between the mother and neonate. We conducted a retrospective study at a tertiary hospital in Japan to investigate the dose-dependent influence of ATDs on both maternal and fetal thyroid hormone status. Materials and Methods: We retrospectively examined 62 pregnant women who delivered between 2007 and 2016
\end{abstract}

karger@karger.com www.karger.com/etj

(C) 2020 European Thyroid Association Published by S. Karger AG, Basel and were treated for Graves' hyperthyroidism with ATD at any stage during pregnancy. We selected individuals whose data on maternal FT4 level within 4 weeks of their deliveries and cord FT4 level of their infants at the time of delivery were available. Those with multiple pregnancies, iodine or glucocorticoid treatment, and fetal goiter detected by ultrasonography were excluded. Results: After the exclusion criteria were applied, we recruited 40 individuals. The cord FT4 levels were significantly lower than the maternal FT4 levels in patients treated with high-dosage ATDs (methimazole $>5$ $\mathrm{mg}$ daily or propylthiouracil $>100 \mathrm{mg}$ daily). However, there were no significant differences between maternal and cord FT4 levels in patients treated with low-dosage ATDs (methimazole $\leq 5 \mathrm{mg}$ daily or propylthiouracil $\leq 100 \mathrm{mg}$ daily). We selected 35 individuals whose data on maternal thyrotropin receptor-binding inhibitory immunoglobulin (TBII) level were available. Multiple linear regression analysis adjusted for ATD dosage, maternal TBII level, and gestational period 
found that ATD dosage was a significant predictor of the difference in serum FT4 levels between the mother and neonate. In terms of maternal complications, multiple logistic regression analysis identified maternal free triiodothyronine (FT3) level as a significant predictor of the incidence of preterm delivery. Conclusions: We found a dose-dependent influence of ATDs on the difference in serum FT4 levels between mothers with Graves' hyperthyroidism and their neonates. Further studies to evaluate the optimal target FT4 and FT3 levels for the mother and neonate during pregnancy may improve the outcome of pregnant women with Graves' hyperthyroidism.

(c) 2020 European Thyroid Association Published by S. Karger AG, Basel
To investigate the effect of different dosages of ATDs on fetal thyroid hormone status, we conducted a retrospective study at a tertiary hospital in Japan. Several guidelines recommended the target maternal FT4 levels to be around the upper limit of the trimester-specific reference range [4-6], which was mainly supported by a retrospective study by Momotani et al. [2]. The authors obtained blood samples from mothers and umbilical cords and compared their FT4 levels. Therefore, we decided to examine the difference in FT4 levels between the maternal and cord blood (calculated as maternal FT4 - cord FT4) to evaluate the influence of ATD dosage.

\section{Materials and Methods}

Antithyroid drugs (ATDs) are the mainstay of medical treatment for Graves' hyperthyroidism, occurring in approximately $0.2 \%$ during pregnancy [1]. All ATDs tend to be more potent in the fetus than in the mother [2-4]. ATD dosage that ensures maternal serum free thyroxine (FT4) levels at or moderately above the upper limit of the reference range is reported to be appropriate for fetal euthyroid status [2]. This recommendation was incorporated into the 2017 American Thyroid Association (ATA) management guideline [4], the 2018 European Thyroid Association (ETA) management guideline [5], and the 2019 Japanese Thyroid Association (JTA) management guideline [6].

There is evidence for the dose-dependent influence of ATDs on fetal thyroid hormone status; specifically, Gardner et al. [3] identified a significant inverse correlation between the cord serum FT4 index and the area under the curve for maternal serum propylthiouracil concentrations. ATD dosage was also reported to inversely relate to the cord FT4 index [7]. In contrast, other studies have identified no significant correlation between the ATD dosage and cord FT4 status [2, 8-10]. These inconsistent results may be explained by methodological differences. Uenaka et al. [11] recently reported that ATD dosage was a significant factor related to neonatal thyroid dysfunction in univariable analysis, but not in multivariable analysis. However, little is known on the dose-dependent influence of ATDs on the difference in FT4 levels between maternal and cord blood in patients with Graves' disease (GD) $[2,9]$. In this connection, future research proposed by the ATA guidelines task force includes a trial assessing the optimal target FT4 level in pregnant women treated for hyperthyroidism [4].

Influence of Antithyroid Drugs on Maternal and Neonatal FT4 Levels

\section{Study Design}

We retrospectively examined 62 pregnant women with GD who delivered at the Seirei Hamamatsu General Hospital between 2007 and 2016 and were treated with ATDs at any stage during pregnancy. The hospital is the largest tertiary hospital in the western part of Shizuoka Prefecture in Japan, where many cases from low-risk to high-risk deliveries are managed [12]. The diagnosis of GD was made before or during pregnancy on the basis of the following criteria: (1) the presence of a goiter, ophthalmopathy, and/ or signs of thyrotoxicosis (e.g., tachycardia), (2) elevated free triiodothyronine (FT3) and/or FT4 levels, (3) suppressed thyroid-stimulating hormone (TSH) level, and (4) a positive thyrotropin receptor-binding inhibitory immunoglobulin (TBII) test [6]. ATDs were given in the lowest possible dosage to maintain maternal FT4 levels in a euthyroid or mildly thyrotoxic state based on the trimester-specific reference range [13].

We selected cases in which the data on maternal FT4 level within 4 weeks of their deliveries and cord FT4 level of their infants at the time of delivery were available. Those who had multiple pregnancies, fetal goiter detected by ultrasonography, iodine or glucocorticoid treatment, and change in ATD after the final evaluation of the maternal thyroid hormone status were excluded. The following information were retrieved from the medical record: maternal age at pregnancy; height at pregnancy; weight at delivery; ATD dosage; maternal serum TSH, FT4, and TBII; the presence of cesarean section, preterm delivery (i.e., delivered before 37 gestational weeks), fetal distress, and postpartum hemorrhage; gestational period; cord serum TSH, FT4, and TBII; neonatal sex; body weight; Apgar scores; and the presence of low birth weight, macrosomia, and congenital abnormalities.

Individuals were classified into 3 groups based on the administration dosage at the time of delivery: no-ATD group (discontinued ATD administration), low-dosage group (propylthiouracil $\leq 100 \mathrm{mg}$ daily or methimazole $\leq 5 \mathrm{mg}$ daily), and high-dosage group (propylthiouracil $>100 \mathrm{mg}$ daily or methimazole $>5 \mathrm{mg}$ daily). Little is known about the dose-dependent influence of ATDs on the difference in thyroid hormone status between the mother and neonate in patients with GD. Momotani et al. [14] reported little relationship between the fetal thyroid hormone status and maternal ATD doses when the dosage of propylthiouracil was clas- 
Table 1. Characteristics of the groups according to different dosages of antithyroid drugs (ATDs; $n=40$ )

\begin{tabular}{|c|c|c|c|c|}
\hline Variables & No-ATD & Low-dosage & High-dosage & $p$ value \\
\hline Number of cases & 17 & 14 & 9 & \\
\hline \multicolumn{5}{|l|}{ Mothers } \\
\hline $\mathrm{Age}^{\mathrm{a}}$ & $32.8 \pm 4.1$ & $32.5 \pm 4.9$ & $32.3 \pm 5.2$ & 0.963 \\
\hline Body mass index ${ }^{a}$ & $24.0 \pm 2.1$ & $24.5 \pm 1.6$ & $26.0 \pm 4.4$ & 0.414 \\
\hline Body weight, $\mathrm{kg}^{\mathrm{a}}$ & $60.8 \pm 6.1$ & $61.7 \pm 6.7$ & $64.9 \pm 10.7$ & 0.902 \\
\hline Dosage of ATDs, mga & 0 & $50.0 \pm 24.0$ & $383.3 \pm 261.0^{* * *, \dagger \dagger \dagger}$ & $<0.001$ \\
\hline $\mathrm{TSH}, \mathrm{mIU} / \mathrm{L}^{\mathrm{b}}$ & $0.77[0.14-1.13]$ & $0.01[0.01-0.55]$ & $0.01[0.01-0.01]^{\dagger \dagger}$ & 0.002 \\
\hline $\mathrm{FT} 4, \mathrm{pmol} / \mathrm{L}^{\mathrm{a}}$ & $13.09 \pm 1.99^{\star}$ & $15.23 \pm 3.08$ & $18.66 \pm 3.83^{* *, \dagger \dagger \dagger}$ & $<0.001$ \\
\hline $\mathrm{FT} 3, \mathrm{pmol} / \mathrm{L}^{\mathrm{b}, \#}$ & $3.87[3.47-4.13]$ & $4.82[3.94-5.84]$ & $5.53[5.44-6.44]^{\dagger \dagger \dagger}$ & $<0.001$ \\
\hline TBII, $\%^{\mathrm{b}, \# \#}$ & $7.1[3.7-12.5]$ & $7.8[4.2-16.5]$ & $30.7[13.4-70.1]^{*, \dagger \dagger}$ & 0.004 \\
\hline Vaginal/cesarean ${ }^{c}$ & $13 / 4$ & $12 / 2$ & $7 / 2$ & 0.800 \\
\hline Gestational period, days $^{\mathrm{a}}$ & $274.6 \pm 9.0$ & $276.8 \pm 10.3$ & $259.4 \pm 18.1^{* *, \dagger \dagger}$ & 0.004 \\
\hline Preterm/full-term delivery ${ }^{\mathrm{c}}$ & $0 / 17$ & $1 / 13$ & $4 / 5^{\dagger \dagger}$ & 0.004 \\
\hline \multicolumn{5}{|l|}{ Cord blood } \\
\hline $\mathrm{TSH}, \mathrm{mIU} / \mathrm{L}^{\mathrm{b}}$ & $3.93[3.17-5.82]^{*}$ & $8.31[5.20-11.69]$ & $10.96[9.75-19.05]^{\dagger \dagger \dagger}$ & 0.001 \\
\hline $\mathrm{FT} 4, \mathrm{pmol} / \mathrm{L}^{\mathrm{a}}$ & $14.93 \pm 1.95$ & $15.32 \pm 3.22$ & $13.40 \pm 5.00$ & 0.008 \\
\hline TSH below RR, FT4 above RR ${ }^{c}$ & $1(5.9 \%)$ & $1(7.1 \%)$ & $1(11.1 \%)$ & 0.888 \\
\hline TSH below RR, FT4 within RR & $1(5.9 \%)$ & $0(0 \%)$ & $0(0 \%)$ & 0.500 \\
\hline TSH within RR, FT4 above $\mathrm{RR}^{\mathrm{c}}$ & $5(29.4 \%)$ & $4(28.6 \%)$ & $1(11.1 \%)$ & 0.550 \\
\hline TSH within RR, FT4 within $\mathrm{RR}^{\mathrm{c}}$ & $10(58.8 \%)$ & $9(64.3 \%)$ & $3(33.3 \%)$ & 0.317 \\
\hline TSH within RR, FT4 below RR ${ }^{c}$ & $0(0 \%)$ & $0(0 \%)$ & $2(22.2 \%)$ & 0.027 \\
\hline TSH above RR, FT4 below RR ${ }^{c}$ & $0(0 \%)$ & $0(0 \%)$ & $2(22.2 \%)$ & 0.027 \\
\hline TBII, \% ${ }^{\mathrm{b}}$ & $5.6[3.3-7.2]$ & $10.5[2.9-13.7]$ & $27.1[14.7-64.1]$ & 0.003 \\
\hline \multicolumn{5}{|c|}{ Difference of values between mother and cord blood } \\
\hline $\mathrm{FT} 4, \mathrm{pmol} / \mathrm{L}^{\mathrm{a}}$ & $-1.84 \pm 2.77$ & $-0.08 \pm 3.87$ & $5.26 \pm 6.07^{* *, \dagger \dagger \dagger}$ & $<0.001$ \\
\hline \multicolumn{5}{|l|}{ Infants } \\
\hline Sex, boy/girl ${ }^{\mathrm{c}}$ & $9 / 8$ & $9 / 5$ & $4 / 5$ & 0.802 \\
\hline Body weight, $\mathrm{g}^{\mathrm{a}}$ & $2,929 \pm 469$ & $3,052 \pm 286$ & $2,735 \pm 611$ & 0.274 \\
\hline Low birth weight ${ }^{c}$ & $3(17.6 \%)$ & $0(0 \%)$ & $2(22.2 \%)$ & 0.203 \\
\hline Apgar score at $1 \min ^{\mathrm{a}}$ & $8.4 \pm 0.5$ & $7.7 \pm 2.0$ & $7.8 \pm 1.1$ & 0.141 \\
\hline Apgar score at $5 \mathrm{~min}^{\mathrm{a}}$ & $9.0 \pm 0.0$ & $8.8 \pm 0.9$ & $8.8 \pm 0.8$ & 0.736 \\
\hline Congenital abnormalities $^{c}$ & $1(5.9 \%)$ & $1(7.1 \%)$ & $1(11.1 \%)$ & 0.888 \\
\hline
\end{tabular}

Significant $p$ values are presented in bold. Continuous variables are expressed as ${ }^{a}$ mean \pm standard deviation or ${ }^{b}$ median with interquartile range in square brackets. The $p$ values were derived using ${ }^{\mathrm{a}}$ one-way analysis of variance, ${ }^{\mathrm{b}}$ Kruskal-Wallis test, or ${ }^{c}$ chi-square test. ${ }^{*} p<0.05,{ }^{* *} p<0.01,{ }^{* * *} p<0.001$ compared with the low-dosage group; ${ }^{\dagger \dagger} p<0.01,{ }^{\dagger \dagger} p<0.001$ compared with the no-ATD group by ${ }^{\mathrm{a}}$ Fisher's least significant difference test, ${ }^{\mathrm{b}}$ Dunnett's multiple comparison test, or ${ }^{\mathrm{c}}$ Fisher's exact test. The dosage of ATDs was calculated as ATD-to-methimazole ratio of 20:1 or ATD-to-propylthiouracil ratio of 1:1. " Maternal free triiodothyronine (FT3) levels in no-ATD, low-dosage, and high-dosage groups were available in 16, 11, and 9 individuals, respectively. ${ }^{\#}$ Maternal thrytropin receptor-binding inhibitory immunoglobulin (TBII) levels in no-ATD, low-dosage, and high-dosage groups were available in 14, 12, and 9 individuals, respectively. FT4, free thyroxine; RR, reference range; TSH, thyroid-stimulating hormone.

sified into the high (>100 mg daily) and low ( $\leq 100 \mathrm{mg}$ ) groups. To clarify the difference between our study and the previous one, we classified propylthiouracil $>100 \mathrm{mg}$ daily into the high-dosage group. ATD dosage was calculated using ratios of 20:1 and 1:1 for methimazole and propylthiouracil, respectively, according to previous studies $[4,15]$.

To predict the dose-dependent influence of ATD on the fetal thyroid hormone status using multiple linear regression analyses, we selected individuals whose data on maternal age, maternal body weight, ATD dosage, maternal FT4 level, maternal TBII level, gestational period, and cord FT4 level were available.

\section{Laboratory Methods}

Serum TSH and FT4 levels were measured using a chemiluminescent immunoassay method (ARCHITECT TSH, ARCHITECT Free T4, and ARCHITECT Free T3; Abbott Corporation, Tokyo, Japan). Reference ranges used at the Seirei Hamamatsu General Hospital were as follows: TSH 0.35-4.94 mIU/L, FT4 9.01-19.05 $\mathrm{pmol} / \mathrm{L}$, and FT3 $2.90-4.90 \mathrm{pmol} / \mathrm{L}$. On the basis of the results of a previous study using the ARCHITECT platform [16], the reference ranges for umbilical cord serum in vaginal deliveries were determined as follows: TSH 2.64-27.32 mIU/L and FT4 10.56$16.01 \mathrm{pmol} / \mathrm{L}$. Those in cesarean deliveries were as follows: TSH 2.32-11.85 mIU/L and FT4 10.95-16.41 pmol/L. TSH values less 




Fig. 1. a Free thyroxine (FT4) levels of mothers and neonates. The $p$ values were derived using a paired $t$ test. b Thyroid-stimulating hormone (TSH) levels of mothers and neonates. The $p$ values were derived using the Wilcoxon rank-sum test. Individuals $(n=40)$ were classified into three groups based on the administration dosage of antithyroid drugs (ATDs) at the time of delivery: no-ATD group (discontinued ATD administration), lowdosage group (propylthiouracil $\leq 100 \mathrm{mg}$ daily or methimazole $\leq 5 \mathrm{mg}$ daily), and high-dosage group (propylthiouracil $>100 \mathrm{mg}$ daily or methimazole $>5 \mathrm{mg}$ daily).

than the minimum detectable level of $0.01 \mathrm{mIU} / \mathrm{mL}$ were defined as $0.01 \mathrm{mIU} / \mathrm{L}$.

TBII was measured by radioimmunoassay (TRAb Cosmic I; Cosmic Corporation, Tokyo, Japan); the value $<10 \%$ was defined as the reference range by the manufacturer.

\section{Statistical Analysis}

Data are expressed as mean \pm standard deviation or median with interquartile range in parentheses. The Kolmogorov-Smirnov tests demonstrated that the frequency distributions of serum FT4 levels in both the maternal and cord blood were not significantly different from a Gaussian distribution, whereas the TSH, FT3, and TBII levels showed a significant difference. One-way analysis of variance followed by Fisher's least significant difference test, Kruskal-Wallis test followed by Dunnett's test, chi-square test, or Fisher's exact test were used for comparisons among multiple ATD dosage categories. The paired $t$ test was used for comparison between the maternal and cord serum FT4 levels. The Wilcoxon rank-sum test was used for comparison between the maternal and cord serum TSH levels. The unpaired $t$ test, Mann-Whitney U test, or Fisher's exact test was used for comparisons between full-term and preterm delivery groups.

To examine how factors including ATD dosage affected the difference in FT4 levels between the maternal and cord blood, regression analyses were conducted before and after adjustment. All variables with $p<0.20$ after simple regression analysis and without collinearity between two variables were investigated using multiple regression analysis. It has been reported that the predictive performance would get worse when strict criteria are applied in the small sample size [17]. We determined $p<0.20$ to select variables for further multiple regression analysis because of the small number of our cases. For preterm delivery, logistic regression analyses were performed to detect predictors with odds ratio before and after adjustment with variables.

All statistical analyses were conducted using the GraphPad PRISM (version 7.0, GraphPad Software, Incorporated, San Di- 
Table 2. Predictors of the difference in free thyroxine (FT4) levels between maternal and cord blood before adjustment $(n=35)$

\begin{tabular}{lrrrr}
\hline Variables & \multicolumn{2}{l}{ Unadjusted } & & \\
\cline { 2 - 5 } & \multicolumn{1}{l}{$B$} & $95 \%$ CI & $\beta$ & $p$ value \\
\hline Age (years) & -0.039 & -0.442 to 0.364 & -0.034 & 0.845 \\
Body weight (kg) & 0.084 & -0.151 to 0.318 & 0.126 & 0.472 \\
Dosage of ATDs (mg) & 0.017 & 0.010 to 0.023 & 0.680 & $<\mathbf{0 . 0 0 1}$ \\
Maternal FT4 (pmol/L) & 1.013 & 0.677 to 1.350 & 0.730 & $<\mathbf{0 . 0 0 1}$ \\
Maternal FT3 (pmol/L) & 1.825 & 0.618 to 3.031 & 0.491 & $\mathbf{0 . 0 0 4}$ \\
Maternal TBII (\%) & 0.105 & 0.032 to 0.178 & 0.453 & $\mathbf{0 . 0 0 6}$ \\
Choice of ATDs (PTU compared to MMI) ${ }^{\# \#}$ & -3.070 & -8.303 to 2.163 & -0.271 & 0.234 \\
Gestational period (days) & -0.095 & -0.216 to 0.027 & -0.266 & 0.122 \\
Term pregnancy* & 2.475 & -2.512 to 7.463 & 0.173 & 0.320 \\
\hline
\end{tabular}

Significant $p$ values are presented in bold. The difference in FT4 levels (pmol/L) between maternal and cord blood was calculated as follows: maternal FT4 - cord FT4. The dosage of antithyroid drugs (ATDs) was calculated as ATD-to-methimazole (MMI) ratio of 20:1 or ATD-to-propylthiouracil (PTU) ratio of 1:1. * Maternal free triiodothyronine (FT3) levels were determined in 32 individuals. ${ }^{\#}$ PTU was administered in 14 individuals, and MMI was administered in 7 individuals. * Preterm delivery compared with full-term delivery. CI, confidence interval; TBII, thyrotropin receptor-binding inhibitory immunoglobulin.

ego, CA, USA) or the BellCurve for Excel (version 3.10, Social Survey Research Information Company, Limited, Tokyo, Japan) software. Statistical significance was defined as $p<0.05$, with 2 -sided $p$ values.

\section{Results}

We recruited 40 individuals after exclusion criteria were applied. The patients' baseline characteristics are summarized in Table 1. Among the different categories of ATD dosage at the time of delivery, significant differences were observed in laboratory tests for thyroid disorders such as maternal TSH, FT4, FT3, and TBII and the cord TSH, FT4, and TBII. The differences in FT4 levels between the mother and the cord blood (calculated as maternal FT4 - cord FT4) were $-1.84 \pm 2.77$, $-0.08 \pm 3.87$, and $5.26 \pm 6.07 \mathrm{pmol} / \mathrm{L}$ in the no-ATD, low-dosage, and high-dosage groups, respectively. The cord FT4 levels were significantly higher than the maternal FT4 levels in the no-ATD group, whereas the cord FT4 levels were significantly lower than the maternal FT4 levels in the high-dosage group (Fig. 1a). Note that there were no significant differences between maternal and cord FT4 levels in the low-dosage group. In contrast to FT4, the cord TSH levels were significantly higher than the maternal TSH levels in all three groups (Fig. 1b).
To examine how factors including ATD dosage affected the difference in FT4 levels between the maternal and cord blood, we selected 35 individuals whose data on maternal age, maternal body weight, maternal FT4 level, maternal TBII level, gestational period, and cord FT4 level were available. The results of simple linear regression analyses of factors related to the difference in FT4 levels between the maternal and cord blood are presented in Table 2. ATD dosage, maternal FT4 level, and maternal TBII level were found to be significant predictors. ATD dosage affects the maternal thyroid hormone levels; therefore, 3 different adjustments were conducted (Table 3). Model 1 multiple linear regression analysis adjusted for ATD dosage, maternal TBII level, and gestational period revealed that ATD dosage was the only significant predictor $(\beta=0.865, p<0.001)$. Model 2 analysis revealed that ATD dosage and maternal FT4 were significant predictors when maternal FT4 level was added to evaluate the effect of ATD dosage to the difference of FT4 levels between the maternal and cord blood. These results indicated that ATD dosage was a significant predictor of the difference in FT4 levels between the maternal and cord blood irrespective of the absence or presence of maternal FT4 as a confounder. Model 3 analysis found that ATD dosage was the only significant predictor when maternal FT3 level was added to Model 1.

Regarding maternal complications, the gestational period in the high-dosage group was $259.4 \pm 18$.1, which was 
Table 3. Predictors of the difference in free thyroxine (FT4) levels between maternal and cord blood after adjustment $(n=35)$

\begin{tabular}{|c|c|c|c|c|c|c|c|c|c|c|c|c|}
\hline \multirow[t]{2}{*}{ Variables } & \multicolumn{4}{|c|}{ Model 1} & \multicolumn{4}{|c|}{ Model 2} & \multicolumn{4}{|l|}{ Model 3} \\
\hline & $B$ & $95 \% \mathrm{CI}$ & $\beta$ & $p$ value & $B$ & $95 \% \mathrm{CI}$ & $\beta$ & $p$ value & $B$ & $95 \% \mathrm{CI}$ & $\beta$ & $p$ value \\
\hline Dosage of ATDs (mg) & 0.021 & 0.011 to 0.032 & 0.865 & $<0.001$ & 0.014 & 0.003 to 0.024 & 0.549 & 0.013 & 0.020 & 0.009 to 0.032 & 0.813 & 0.001 \\
\hline Maternal FT4 (pmol/L) & & & & & 0.804 & 0.321 to 1.286 & 0.579 & 0.002 & & & & \\
\hline Maternal FT3 $(\mathrm{pmol} / \mathrm{L})^{\#}$ & & & & & & & & & 0.699 & -0.824 to 2.222 & 0.188 & 0.355 \\
\hline Maternal TBII (\%) & -0.054 & -0.155 to 0.048 & -0.232 & 0.291 & -0.088 & -0.179 to 0.002 & -0.381 & 0.055 & -0.064 & -0.177 to 0.048 & -0.277 & 0.251 \\
\hline Gestational period (days) & 0.001 & -0.104 to 0.107 & 0.003 & 0.982 & -0.005 & -0.096 to 0.086 & -0.014 & 0.912 & 0.014 & -0.106 to 0.134 & 0.039 & 0.812 \\
\hline
\end{tabular}

Significant $p$ values are presented in bold. The difference in FT4 levels (pmol/L) between maternal and cord blood was calculated as follows: maternal FT4 - cord FT4. The dosage of antithyroid drugs (ATDs) was calculated as ATD-to-methimazole ratio of 20:1 or ATD-to-propylthiouracil ratio of 1:1. ${ }^{\#}$ Maternal free triiodothyronine (FT3) levels were available in 32 individuals. Model 1 adjusted analysis: dosage of ATDs, maternal thyrotropin receptor-binding inhibitory immunoglobulin (TBII), and gestational period. Model 2 adjusted analysis: dosage of ATDs, maternal FT4, maternal TBII, and gestational period. Model 3 adjusted analysis: dosage of ATDs, maternal FT3, maternal TBII, and gestational period. CI, confidence interval.

Table 4. Characteristics in the full-term and preterm delivery groups $(n=40)$

\begin{tabular}{|c|c|c|c|}
\hline Variables & Full-term delivery & Preterm delivery & $p$ values \\
\hline Number of cases & 35 & 5 & \\
\hline \multicolumn{4}{|l|}{ Mothers } \\
\hline Age $^{\mathrm{a}}$ & $32.5 \pm 4.5$ & $33.6 \pm 5.0$ & 0.603 \\
\hline Body mass index ${ }^{\mathrm{a}}$ & $24.4 \pm 2.0$ & $26.3 \pm 5.8$ & 0.137 \\
\hline Body weight, $\mathrm{kg}^{\mathrm{a}}$ & $61.6 \pm 6.2$ & $64.9 \pm 14.3$ & 0.363 \\
\hline Dosage of ATDs, $\mathrm{mg}^{\mathrm{a}}$ & $70.0 \pm 143.8$ & $340.0 \pm 338.0$ & 0.003 \\
\hline $\mathrm{TSH}, \mathrm{mIU} / \mathrm{L}^{\mathrm{b}}$ & $0.14[0.01-0.84]$ & $0.01[0.01-0.01]$ & 0.062 \\
\hline $\mathrm{FT} 4, \mathrm{pmol} / \mathrm{L}^{\mathrm{a}}$ & $14.67 \pm 3.30$ & $18.07 \pm 4.09$ & 0.043 \\
\hline $\mathrm{FT} 3, \mathrm{pmol} / \mathrm{L}^{\mathrm{b}, \#}$ & $4.08[3.69-5.17]$ & $7.16[6.14-7.27]$ & $<0.001$ \\
\hline TBII, \% ${ }^{\mathrm{b}, \# \#}$ & $9.0[4.3-14.6]$ & $28.3[19.2-71.1]$ & 0.012 \\
\hline Vaginal/cesarean $^{c}$ & $27 / 8$ & $5 / 0$ & 0.563 \\
\hline Gestational period, days ${ }^{\mathrm{a}}$ & $276.1 \pm 7.7$ & $243.2 \pm 9.7$ & $<0.001$ \\
\hline \multicolumn{4}{|l|}{ Cord blood } \\
\hline $\mathrm{TSH}, \mathrm{mIU} / \mathrm{L}^{\mathrm{b}}$ & $5.82[3.90-9.37]$ & $10.96[9.34-67.52]$ & 0.145 \\
\hline $\mathrm{FT} 4, \mathrm{pmol} / \mathrm{L}^{\mathrm{a}}$ & $14.63 \pm 2.35$ & $15.37 \pm 7.54$ & 0.643 \\
\hline TBII, $\%{ }^{\mathrm{b}}$ & $6.8[3.6-12.7]$ & $14.7[5.0-64.1]$ & 0.341 \\
\hline \multicolumn{4}{|c|}{ Difference of values between mother and cord blood } \\
\hline FT4, $\mathrm{pmol} / \mathrm{L}^{\mathrm{a}}$ & $0.04 \pm 4.02$ & $2.70 \pm 9.15$ & 0.255 \\
\hline \multicolumn{4}{|l|}{ Infants } \\
\hline Sex, boy/girl ${ }^{c}$ & $18 / 17$ & $4 / 1$ & 0.356 \\
\hline Body weight, $\mathrm{g}^{\mathrm{a}}$ & $2,999 \pm 388$ & $2,436 \pm 636$ & 0.008 \\
\hline Low birth weight ${ }^{\mathrm{c}}$ & $3(8.6 \%)$ & $2(40.0 \%)$ & 0.109 \\
\hline Apgar score at $1 \min ^{\mathrm{a}}$ & $8.3 \pm 0.5$ & $6.2 \pm 3.3$ & $<0.001$ \\
\hline Apgar score at $5 \mathrm{~min}^{\mathrm{a}}$ & $9.0 \pm 0.3$ & $8.0 \pm 1.4$ & $<0.001$ \\
\hline Congenital abnormalities ${ }^{c}$ & $2(5.7 \%)$ & $1(20.0 \%)$ & 0.338 \\
\hline
\end{tabular}

Significant $p$ values are presented in bold. Continuous variables are expressed as ${ }^{a}$ mean \pm standard deviation or ${ }^{c}$ median with interquartile range in square brackets. The $p$ values were derived using ${ }^{\mathrm{a}}$ unpaired $t$ test, ${ }^{\mathrm{b}}$ MannWhitney U test, or ${ }^{c}$ Fisher's exact test. The dosage of antithyroid drugs (ATDs) was calculated as ATD-to-methimazole ratio of 20:1 or ATD-to-propylthiouracil ratio of 1:1. \# Maternal free triiodothyronine (FT3) levels in full-term delivery were available in 31 individuals. ${ }^{\#}$ Maternal thyrotropin receptor-binding inhibitory immunoglobulin (TBII) levels in full-term delivery were available in 30 individuals. FT4, free thyroxine; TSH, thyroidstimulating hormone.

Influence of Antithyroid Drugs on Maternal and Neonatal FT4 Levels
Eur Thyroid J 2021;10:372-381

DOI: $10.1159 / 000509324$ 
Table 5. Predictors of preterm delivery before and after adjustment $(n=35)$

\begin{tabular}{|c|c|c|c|c|c|c|c|c|c|}
\hline Variables & \multicolumn{3}{|l|}{ Unadjusted } & \multicolumn{3}{|l|}{ Adjusted $^{1}$} & \multicolumn{3}{|l|}{ Adjusted $^{2}$} \\
\hline Body weight $(\mathrm{kg})$ & 1.054 & 0.941 to 1.182 & 0.361 & & & & & & \\
\hline Dosage of ATDs (mg) & 1.005 & 1.001 to 1.009 & 0.038 & 1.003 & 0.995 to 1.012 & 0.471 & 1.001 & 0.992 to 1.010 & 0.828 \\
\hline Maternal FT4 (pmol/L) & 1.249 & 0.967 to 1.613 & 0.089 & 0.884 & 0.524 to 1.492 & 0.645 & & & \\
\hline
\end{tabular}

Significant $p$ values are presented in bold. The dosage of antithyroid drugs (ATDs) was calculated as ATD-to-methimazole ratio of 20:1 or ATD-topropylthiouracil ratio of 1:1. ${ }^{\#}$ Maternal free triiodothyronine (FT3) levels were available in 32 individuals. ${ }^{1}$ Adjusted analysis: dosage of ATDs, maternal free thyroxine (FT4), and maternal TBII. ${ }^{2}$ Adjusted analysis: dosage of ATDs, maternal FT3, and maternal TBII. CI, confidence interval.

significantly shorter than that in the no-ATD $(276.6 \pm 9.0)$ and low-dosage groups $(276.8 \pm 10.3$; Table 1$)$. In line with this, the incidence of preterm delivery in the high-dosage group was most frequent among the three groups. When comparing the characteristics in the full-term delivery with those in the preterm delivery, significant differences were observed in ATD dosage and maternal FT4, FT3, and TBII (Table 4). Subsequent simple logistic regression analyses revealed that ATD dosage and maternal FT3 and TBII levels were significant predictors of preterm delivery. Multiple logistic regression analysis adjusted for ATD dosage, maternal FT4, and maternal TBII identified no significant predictors, whereas multivariable analysis adjusted for ATD dosage, maternal FT3, and maternal TBII revealed that maternal FT3 level was the only significant predictor (odds ratio, 5.794; $p=0.028$; Table 5). With regard to neonatal sex, body weight, Apgar scores, and the presence of low birth weight and congenital abnormalities, there were no significant differences among the three groups. Fetal distress, postpartum hemorrhage, and macrosomia were not identified in this study.

\section{Discussion}

We retrospectively examined pregnant women with GD at a tertiary hospital in Japan and found a dose-dependent influence of ATDs on the difference in serum FT4 levels between the mother and neonate (Tables 1, 3). The strength of our study lies in the premise that confounding factors of fetal thyroid function were taken into consideration other than the ATD dosage.

Thyroid hormones are critical for the normal development of the central nervous system. In several recent studies, even mild maternal thyroid hormone insufficiency during the perinatal period was revealed to be associated with a potential risk of brain development impairment [18]. Evaluation of the fetal thyroid status can be achieved through a combination of imaging modalities, such as ultrasonography and magnetic resonance imaging, cordocentesis, and amniocentesis [19]. However, each method has an important shortcoming. Imaging modalities have a limited ability to detect slight fetal thyroid dysfunctions without goiter formation. Cordocentesis, the gold standard diagnostic method to evaluate the fetal thyroid hormone status, is invasive and technically demanding. Amniotic fluid sampling is safer than cordocentesis, but the thyroid hormone status in the amniotic fluid may not accurately reflect the fetal thyroid hormone status. In contrast to these methods, measuring the maternal thyroid hormone status is currently a convenient and reliable approach to monitor the fetal thyroid hormone status $[4-6,10]$.

The fetus is dependent on the transplacental passage of maternal supply of thyroid hormones before the onset of fetal thyroid function during mid-gestation [10]. Once the fetal thyroid gland becomes functional, TSH-receptor autoantibodies, such as thyroid-stimulating antibodies and thyroid-blocking antibodies that are able to cross the placenta, affect the fetal thyroid gland in pregnant women with GD. Mortimer and coworkers [7] reported that the maternal TBII at delivery was well correlated with maternal cord TBII (Pearson's $r$ value, $0.940 ; p<0.001$ ). ATDs given to the mother also cross the placenta, but the fetal thyroid responds more strongly to ATD therapy than the maternal thyroid. This could be the reason why ATD dosages sufficient to maintain mothers in a euthyroid state often induce fetal hypothyroidism [4]. 
The ETA, ATA, and JTA guidelines have recommended the lowest effective dose of ATDs to maintain maternal serum FT4 levels at (or just above) the upper limit of the trimester-specific reference range [4-6], which was supported by the previous study by Momotani et al. [2]. By selecting 43 pregnant women with GD who were treated with ATDs at the time of delivery, the authors found that serum FT4 levels were lower in the cord bloods than in the maternal bloods. However, ATD dosage was not taken into consideration in the ETA, ATA, and JTA guidelines. Thus, we evaluated whether ATD dosage affects the difference in FT4 levels between the mother and neonate. To the best of our knowledge, this is the first study of its kind.

Importantly, there were no significant differences between the maternal and cord FT4 levels in the low-dosage group (Fig. 1). It is possible that the fetus may be undertreated, when the maternal FT4 levels are set around the upper limit of the reference range treated with low-dosage ATDs. The undertreatment of maternal hyperthyroidism has been reported to result in central congenital hypothyroidism [20]. Accordingly, we propose to replace the present statement in the guidelines with the following sentences: "Targeting maternal FT4 levels should not exceed the upper limit of the trimester-specific reference range when administering low dosage of ATDs (propylthiouracil $\leq 100 \mathrm{mg}$ daily or methimazole $\leq 5 \mathrm{mg}$ daily). Targeting maternal FT4 levels should be at or just above the upper limit of reference range according to ATD dosage when administering high dosage of ATDs (propylthiouracil $>100 \mathrm{mg}$ daily or methimazole $>5 \mathrm{mg}$ daily)."

The small sample size is a weakness of our study. Guidelines have recommended being stably euthyroid before attempting pregnancy; hence, it seems to be difficult to recruit pregnant patients with GD treated with high-dosage ATDs. A large-scale, multi-institutional joint research that evaluates optimal target FT4 levels based on the different ATD dosages would be required to provide enough high-quality evidence to modify the statements in the present guidelines.

Of additional interest is the finding that the standard deviation of the differences in FT4 levels between the mother and cord blood was 2.77, 3.87, and $6.07 \mathrm{pmol} / \mathrm{L}$ in the no-ATD, low-dosage, and high-dosage groups, respectively (Table 1). Although mean maternal FT4 levels were highest in the high-dosage group, note that cord thyroid hormone status in this group was overt hypothyroidism in 2 of 9 neonates. In the current guidelines, ATD administration at the lowest effective dose is recommended to minimize adverse effects. This recommendation may also be supported by our findings in which the discrepancy between the mother and cord FT4 levels seemed to be ATD dose dependent. We also propose that ATD dosage should be reduced as much as possible using adequate treatment before women with GD attempt pregnancy.

Thionamides that are most frequently used during pregnancy are propylthiouracil and methimazole [2]. In 1977 , an in vivo study found that the human placenta was more permeable to methimazole than to propylthiouracil [21]; however, a subsequent in vitro study revealed that both drugs had similar placental transfer kinetics [22]. Furthermore, other studies have indicated that these two drugs are comparable in GD treatment at least in terms of fetal hypothyroidism-inducing potential $[7,14]$. In this connection, our study added the additional evidence that propylthiouracil and methimazole are comparable even in terms of the influence on the difference in FT4 levels between maternal and cord blood (Table 2).

Thyroid function should be controlled not only in the fetus but also in the mother [23]. Moreover, poor control of thyrotoxicosis is associated with several maternal disorders including preeclampsia, congestive heart failure, and preterm delivery [4]. For example, Millar et al. [24] reported that the risk of preterm delivery was significantly higher (odds ratio, 16.5) among women who had hyperthyroidism at presentation and delivery compared with women who had euthyroidism during pregnancy. In agreement with the previous study, our multiple logistic regression analysis identified the maternal FT3 level as a significant predictor of the incidence of preterm delivery. These findings suggest that undertreatment should be avoided in terms of maternal complications. Further studies are needed to assess the optimal maternal FT3 level in addition to FT4 level that is appropriate for both mothers and neonates.

This study has the following limitations. First, its retrospective design has the potential for introducing biases. Second, the maximum duration from maternal blood sampling to cord blood sampling was 4 weeks, which cannot exclude the possible changes of maternal thyroid hormone in this period. In addition, pregnancy is associated with significant and reversible changes influencing the thyroid hormone status. For example, maternal serum thyroxine-binding globulin concentrations increase during the first half of gestation [25]. Thus, our findings may not apply to other periods during pregnancy. Third, reference intervals of thyroid function tests from the umbilical cord blood were not established in our hospital, although the reference ranges for umbilical cord serum 
have been reported to be affected by several factors including lifestyle and other environmental factors for every population in different regions even within a country [26]. Last, the possible discrepancy of ATD dosage between the medical record and actual consumption cannot be excluded because patient medication compliance was not monitored.

In conclusion, we analyzed 40 pregnant women with Graves' hyperthyroidism and obtained several important findings. First, the dose-dependent effect of ATDs on the difference in FT4 levels between the mother and neonate was confirmed in patients with GD. Particularly, no significant differences between maternal and cord FT4 levels were observed in the low-dosage group. On the basis of these findings, ATD dosage that ensures FT4 levels at or just above the upper limit of the trimester-specific reference range may not be appropriate when administering low-dosage ATDs. Second, the variation in the discrepancy between maternal and cord FT4 levels was smaller in the low-dosage group than in the high-dosage group, suggesting that minimizing the use of ATDs before attempting pregnancy would be recommended in terms of controlling FT4 levels. Third, maternal FT3 level was identified as an independent predictor of the incidence of preterm delivery. Further studies to assess the optimal maternal target FT4 and FT3 levels for both the mother and neonate during pregnancy may improve the outcome in pregnant women with Graves' hyperthyroidism.

\section{Acknowledgments}

The authors are grateful to Dr. Naoko Arata for her valuable comments. Portions of the manuscript were presented at the Annual Meeting of the American Thyroid Association held in Chicago, 2019.

\section{Statement of Ethics}

The study protocol was approved by the Institutional Review Boards of Seirei Hamamatsu General Hospital (No. 2264) and was congruent with the Declaration of Helsinki. Written informed consent was waived because of the retrospective design.

\section{Conflict of Interest Statement}

The authors have no conflicts of interest to declare.

\section{Funding Sources}

No specific funding was obtained for the present study.

\section{Author Contributions}

H.I. designed the study, collected and analyzed data, and wrote the paper. K.O. analyzed data, wrote the paper, and organized the study. E.O. analyzed data and wrote the paper. T.M., Y.K., C.H., A.M., and S.S. collected and analyzed data. T.S. and Y.O. analyzed data and revised the manuscript. R.G. designed the study, collected and analyzed data, and revised the manuscript.

\section{References}

1 Cooper DS, Laurberg P. Hyperthyroidism in pregnancy. Lancet Diabetes Endocrinol. 2013 Nov; 1(3):238-49.

2 Momotani N, Noh J, Oyanagi H, Ishikawa N, Ito K. Antithyroid drug therapy for Graves' disease during pregnancy. Optimal regimen for fetal thyroid status. N Engl J Med. 1986 Jul; 315(1):24-8.

3 Gardner DF, Cruikshank DP, Hays PM, Cooper DS. Pharmacology of propylthiouracil (PTU) in pregnant hyperthyroid women: correlation of maternal PTU concentrations with cord serum thyroid function tests. J Clin Endocrinol Metab. 1986 Jan;62(1):217-20.

4 Alexander EK, Pearce EN, Brent GA, Brown RS, Chen H, Dosiou C, et al. 2017 Guidelines of the American Thyroid Association for the diagnosis and management of thyroid disease during pregnancy and the postpartum. Thyroid. 2017 Mar;27(3):315-89.

5 Kahaly GJ, Bartalena L, Hegedüs L, Leenhardt L, Poppe K, Pearce SH. 2018 European Thy- roid Association guideline for the management of Graves' hyperthyroidism. Eur Thyroid J. 2018 Aug;7(4):167-86.

6 The Japan Thyroid Association. Guideline for the treatment of Graves' disease in Japan. Tokyo: Nankodo; 2019 (in Japanese).

7 Mortimer RH, Tyack SA, Galligan JP, PerryKeene DA, Tan YM. Graves' disease in pregnancy: TSH receptor binding inhibiting immunoglobulins and maternal and neonatal thyroid function. Clin Endocrinol (Oxf). 1990 Feb;32(2):141-52.

8 Cheron RG, Kaplan MM, Larsen PR, Selenkow HA, Crigler JF Jr. Neonatal thyroid function after propylthiouracil therapy for maternal Graves' disease. N Engl J Med. 1981 Feb; 304(9):525-8.

9 Momotani N, Yamashita R, Yoshimoto M, Noh J, Ishikawa N, Ito K. Recovery from foetal hypothyroidism: evidence for the safety of breast-feeding while taking propylthiouracil. Clin Endocrinol (Oxf). 1989 Nov;31(5):591-5.
10 Bliddal S, Rasmussen AK, Sundberg K, Brocks V, Feldt-Rasmussen U. Antithyroid drug-induced fetal goitrous hypothyroidism. Nat Rev Endocrinol. 2011 Mar;7(7):396-406.

11 Uenaka M, Tanimura K, Tairaku S, Morioka I, Ebina Y, Yamada H. Risk factors for neonatal thyroid dysfunction in pregnancies complicated by Graves' disease. Eur J Obstet Gynecol Reprod Biol. 2014 Jun;177:89-93.

12 Yorifuji T, Naruse H, Kashima S, Murakoshi $\mathrm{T}$, Kato $\mathrm{T}$, Inoue $\mathrm{S}$, et al. Trends of preterm birth and low birth weight in Japan: a one hospital-based study. BMC Pregnancy Childbirth. 2012 Dec;12(1):162.

13 Kurioka H, Takahashi K, Miyazaki K. Maternal thyroid function during pregnancy and puerperal period. Endocr J. 2005 Oct;52(5):587-91.

14 Momotani N, Noh JY, Ishikawa N, Ito K. Effects of propylthiouracil and methimazole on fetal thyroid status in mothers with Graves' hyperthyroidism. J Clin Endocrinol Metab. 1997 Nov;82(11):3633-6. 
15 Nicholas WC, Fischer RG, Stevenson RA, Bass JD. Single daily dose of methimazole compared to every 8 hours propylthiouracil in the treatment of hyperthyroidism. South Med J. 1995 Sep;88(9):973-6.

16 Fan P, Luo ZC, Tang N, Wang W, Liu Z, Zhang J, et al. Advanced Maternal Age, Mode of Delivery, and Thyroid Hormone Levels in Chinese Newborns. Front Endocrinol (Lausanne). 2020 Jan; 10:913.

17 Steyerberg EW. Clinical prediction models: a practical approach to development, validation, and updating. New York: Springer; 2009.

18 Thompson W, Russell G, Baragwanath G, Matthews J, Vaidya B, Thompson-Coon J. Maternal thyroid hormone insufficiency during pregnancy and risk of neurodevelopmental disorders in offspring: A systematic review and meta-analysis. Clin Endocrinol (Oxf). 2018 Apr;88(4):575-84.
19 Iijima S. Current knowledge about the in utero and peripartum management of fetal goiter associated with maternal Graves' disease. Eur J Obstet Gynecol Reprod Biol X. 2019 May;3: 100027.

20 De Groot L, Abalovich M, Alexander EK, Amino N, Barbour L, Cobin RH, et al. Management of thyroid dysfunction during pregnancy and postpartum: an Endocrine Society clinical practice guideline. J Clin Endocrinol Metab. 2012 Aug;97(8):2543-65.

21 Marchant B, Brownlie BE, Hart DM, Horton PW, Alexander WD. The placental transfer of propylthiouracil, methimazole and carbimazole. J Clin Endocrinol Metab. 1977 Dec; 45(6):1187-93.

22 Mortimer RH, Cannell GR, Addison RS, Johnson LP, Roberts MS, Bernus I. Methimazole and propylthiouracil equally cross the perfused human term placental lobule. J Clin Endocrinol Metab. 1997 Sep;82(9):3099-102.
23 Laurberg P, Bournaud C, Karmisholt J, Orgiazzi J. Management of Graves' hyperthyroidism in pregnancy: focus on both maternal and foetal thyroid function, and caution against surgical thyroidectomy in pregnancy. Eur J Endocrinol. 2009 Jan;160(1):1-8.

24 Millar LK, Wing DA, Leung AS, Koonings PP, Montoro MN, Mestman JH. Low birth weight and preeclampsia in pregnancies complicated by hyperthyroidism. Obstet Gynecol. 1994 Dec;84(6):946-9.

25 Krassas GE, Poppe K, Glinoer D. Thyroid function and human reproductive health. Endocr Rev. 2010 Oct;31(5):702-55.

26 Mehari A, Challa F, Gebreyesus G, Alemayehu D, Seifu D. Establishment of reference intervals of thyroid function tests from cord blood of neonates in two selected hospitals, Addis Ababa, Ethiopia. BMC Pediatr. 2016 Aug;16(1):118. 